\title{
A Refinement of the Millionshchikov Quasi-Normality Hypothesis for Convective Boundary Layer Turbulence
}

\author{
VLADIMIR M. GRYANIK \\ Alfred Wegener Institute for Polar and Marine Research, Bremerhaven, Germany, and A. M. Obukhov Institute of Atmospheric \\ Physics, Russian Academy of Sciences, Moscow, Russia \\ JÖRG HARTMANN \\ Alfred Wegener Institute for Polar and Marine Research, Bremerhaven, Germany \\ Siegfried RAASCH AND Michael SCHRÖTER \\ University of Hannover, Hannover, Germany
}

(Manuscript received 26 July 2004, in final form 29 October 2004)

\begin{abstract}
The Millionshchikov hypothesis of quasi-normal distribution of fourth-order moments fails for convective conditions where the probability density functions of temperature and vertical velocity fluctuations are skewed. This is shown for aircraft and large-eddy simulation (LES) data, and new closures for fourth-order moments that take the skewness into account are suggested. These new closures are in very good agreement with the data.
\end{abstract}

\section{Introduction}

The Millionshchikov hypothesis (Millionshchikov 1941; Monin and Yaglom 1971) states that in higherorder turbulence closure (HOC) models, the fourthorder moments (FOM) can be approximated as quasinormal (Gaussian) even if the third-order moments (TOM) are nonzero. For a long time, this hypothesis was adopted without discussion in a large number of turbulence closure models (e.g., Andre et al. 1976; Moeng and Randall 1984; Canuto et al. 1994). Recent theoretical studies (e.g., Ilyushin and Kurbatskii 1997; Canuto et al. 2001; Gryanik and Hartmann 2002, hereafter $\mathrm{GH}$ ) and analyses of measurements (e.g., GH; Alberghi et al. 2002) indicate that the Millionshchikov hypothesis fails for vertical velocity and temperature in convective boundary layer (CBL) conditions. Furthermore, we have for the first time FOMs from large-eddy simulation (LES) data available. They also show that the Millionshchikov hypothesis fails for the same con-

Corresponding author address: Dr. Jörg Hartmann, Alfred Wegener Institute for Polar and Marine Research, Postfach 12 0161, Bremerhaven 27515, Germany.

E-mail: jhartmann@awi-bremerhaven.de ditions. Losch (2004) found very similar behavior of the FOMS in open-ocean deep convection. In this note, we present a refinement of the Millionshchikov hypothesis as applied to CBL conditions.

Using aircraft measurements and LES data we show that the FOM in vertical velocity and temperature are essentially non-Gaussian, while the FOM in the horizontal along and across wind components are close to Gaussian.

In GH, we explained the shortcoming of the Millionshchikov hypothesis by the skewed nature of CBL turbulence with respect to upward-downward and hot-cold fluctuations and derived non-Gaussian FOM parameterizations for $\overline{w^{\prime 4}}, \overline{w^{\prime 3} \theta^{\prime}}, \overline{w^{\prime} \theta^{\prime 3}}$, and $\overline{\theta^{\prime 4}}$ (here, $w^{\prime}$ is the vertical velocity fluctuation and $\theta^{\prime}$ the temperature fluctuations), which explicitly account for the effects of asymmetry between upward and downward motion and also between warm and cold areas of the fluid by semiorganized coherent structures. Here we extend the twoscale top-hat method of $\mathrm{GH}$ to also include the horizontal velocity components leading to a 16-delta model of the probability density function (PDF). We test these parameterizations of GH against a new set of LES data, and suggest new non-Gaussian FOM parameterizations for the other moments not yet considered but explicitly 
involved into HOC models $\overline{w^{\prime 2} \theta^{\prime 2}}, \overline{u^{\prime 4}}, \overline{v^{\prime 4}}, \overline{w^{\prime 2} u^{\prime 2}}$, $\overline{w^{\prime 2} v^{\prime 2}}, \overline{w^{\prime 2} q^{\prime 2}}$ (here, $u^{\prime}, v^{\prime}$ are the along-wind and crosswind horizontal velocity components, $q^{\prime 2}=u^{\prime 2}+v^{\prime 2}+$ $\left.w^{\prime 2}\right)$. For all the moments considered, the agreement between the parameterizations proposed and the data is very good.

\section{Testing of the Millionshchikov hypothesis versus data and LES}

The aircraft measurements are obtained during the Arctic Radiation and Turbulence Interaction Study (ARTIST) campaign (Hartmann et al. 1999) in a convectively driven boundary layer over the ocean with 8-12 $\mathrm{m} \mathrm{s}^{-1}$ wind speed, further details can also be found in GH. The LES data are from a simulation of a convective boundary layer with $12 \mathrm{~m} \mathrm{~s}^{-1}$ geostrophic wind speed, they are described in detail in Raasch and Schröter (2001). In Fig. 1 the fourth order moments are plotted versus their Gaussian parameterizations (Monin and Yaglom 1971)

$$
\overline{a^{\prime} b^{\prime} c^{\prime} d^{\prime}}=\overline{a^{\prime} b^{\prime}} \cdot \overline{c^{\prime} d^{\prime}}+\overline{a^{\prime} c^{\prime}} \cdot \overline{b^{\prime} d^{\prime}}+\overline{a^{\prime} d^{\prime}} \cdot \overline{b^{\prime} c^{\prime}},
$$

where $a^{\prime}, b^{\prime}, c^{\prime}, d^{\prime}$ are the fluctuations $w^{\prime}, u^{\prime}, v^{\prime}$, and $\theta^{\prime}$. Since the horizontal velocity components have a very small skewness, the FOM of the along-wind $\overline{u^{\prime 4}}$ and cross-wind $\overline{v^{\prime 4}}$ horizontal velocities agree with the Millionshchikov hypothesis both for measurements and LES data.

All fourth-order moments involving the vertical velocity component or the temperature mostly are significantly larger than their Gaussian prediction. The Gaussian prediction gives an estimation of a lower bound for all these moments, except for the FOM of the across-wind component $\overline{v^{\prime 4}}$. As an indication for the suitability of the parameterization we calculate the explained variances $\sigma_{f}^{2}=1-\overline{\left(y_{i}-f\left(x_{i}\right)\right)^{2}} / \overline{\left(y_{i}-\bar{y}\right)^{2}}$, where $y_{i}$ are the aircraft measurements and LES data, plotted at the ordinate, and $f\left(x_{i}\right)$ the parameterizations (abscissas). For the vertical wind component of the LES data, the Gaussian parameterization $3{\overline{w^{\prime 2}}}^{2}$ only explains $68 \%$ of the variance of $\overline{w^{\prime 4}}$, and for the aircraft data $67 \%$. The agreement is worse for some cross moments for the aircraft data since measurement accuracy also contributes to the unexplained variance, see also GH. The LES data do not suffer such errors and lead to higher values of the explained variance. The LES data are plotted as a continuous line in the order of increasing height. The loops in these plots, especially for the moments $\overline{w^{\prime 4}}$, $\overline{w^{\prime 3} \theta^{\prime}}$ and $\overline{w^{\prime 2} \theta^{\prime 2}}$, represent an ambiguity since the relation is not constant with height. An ambiguity like this is a serious drawback of any parameterization.

\section{New expressions for FOM}

Gryanik and Hartmann (2002) assumed that a measure of deviation of statistics from the Gaussian behavior is provided by the skewness $\left(S_{x}=\left(\overline{x^{\prime 3}}\right) /\left[\overline{x^{\prime 2}}(3 / 2)\right)\right]$ of vertical velocity $S_{w}$ and temperature $S_{\theta}$, and suggested the parameterizations

$$
\begin{aligned}
\overline{w^{\prime 4}} & =a_{3}\left(1+d_{3} S_{w}^{2}\right){\overline{w^{\prime 2}}}^{2}, \\
\overline{\theta^{\prime 4}} & =a_{4}\left(1+d_{4} S_{\theta}^{2}\right){\overline{\theta^{\prime 2}}}^{2}, \\
\overline{w^{\prime 3} \theta^{\prime}} & =a_{5}\left(1+d_{5} S_{w}^{2}\right) \overline{w^{\prime 2}} \overline{w^{\prime} \theta^{\prime}} \\
& =a_{5}\left(1+d_{5} S_{w}^{2}\right) C_{w \theta^{w^{\prime 2}}}{ }^{3 / 2} \overline{\theta^{\prime 2}}{ }^{1 / 2}, \\
\overline{w^{\prime} \theta^{\prime 3}} & =a_{6}\left(1+d_{6} S_{\theta}^{2}\right) \overline{\theta^{\prime 2}} \overline{w^{\prime} \theta^{\prime}} \\
& =a_{6}\left(1+d_{6} S_{\theta}^{2}\right) C_{w \theta^{w^{\prime 2}}}{\overline{\theta^{\prime}}}^{{\theta^{\prime 2}}^{3 / 2}},
\end{aligned}
$$

where

$$
C_{w \theta}=\frac{\overline{w^{\prime} \theta^{\prime}}}{{\overline{w^{\prime 2}}}^{1 / 2}{\overline{\theta^{\prime 2}}}^{1 / 2}}
$$

is the correlation coefficients of $w$ and $\theta$, and $a_{i}(i=3$, $\ldots, 11)$ and $d_{i}(i=3, \ldots, 11)$ are dimensionless parameters. Equations (2)-(5) are discussed in GH in detail and will here be tested against the new LES data of low-to-moderate wind.

If we, in analogy to $\mathrm{GH}$, assume the skewness of the along-wind $S_{u}$ and cross-wind $S_{v}$ horizontal velocity components to be a measure of the deviation of statistics from the Gaussian behavior, we suggest generalizing the Millionshchikov hypothesis to the new FOM closure of the following form:

$$
\begin{aligned}
& \overline{w^{\prime 2} \theta^{\prime 2}}=a_{7}\left[\left(\overline{w^{\prime 2}} \overline{\theta^{\prime 2}}+2{\overline{w^{\prime} \theta^{\prime}}}^{2}\right)+d_{7} S_{w} S_{\theta^{\prime}} \overline{w^{\prime} \theta^{\prime}}{\overline{w^{\prime 2}}}^{1 / 2}{\overline{\theta^{\prime 2}}}^{1 / 2}\right] \\
& =a_{7}\left(1+d_{7} \frac{C_{w \theta}}{1+2 C_{w \theta}^{2}} S_{w} S_{\theta}\right)\left(\overline{w^{\prime 2}} \overline{\theta^{\prime 2}}+2{\overline{w^{\prime} \theta^{\prime}}}^{2}\right), \\
& \overline{u^{\prime 4}}=a_{8}\left(1+d_{8} S_{u}^{2}\right){\overline{u^{\prime 2}}}^{2}, \\
& \overline{v^{\prime 4}}=a_{9}\left(1+d_{9} S_{v}^{2}\right){\overline{v^{\prime 2}}}^{2}, \\
& \overline{w^{\prime 2} u^{\prime 2}}=a_{10}\left(1+d_{10} \frac{C_{w u}}{1+2 C_{w u}^{2}} S_{w} S_{u}\right) \\
& \times\left(\overline{w^{\prime 2}} \overline{u^{\prime 2}}+2{\overline{w^{\prime} u^{\prime}}}^{2}\right) \\
& \left.\overline{w^{\prime 2} v^{\prime 2}}=a_{11}\left(1+d_{11} \frac{C_{w v}}{1+2 C_{w v}^{2}} S_{w} S_{v}\right) \overline{\left(w^{\prime 2}\right.} \overline{v^{\prime 2}}+2{\overline{w^{\prime} v^{\prime}}}^{2}\right),
\end{aligned}
$$



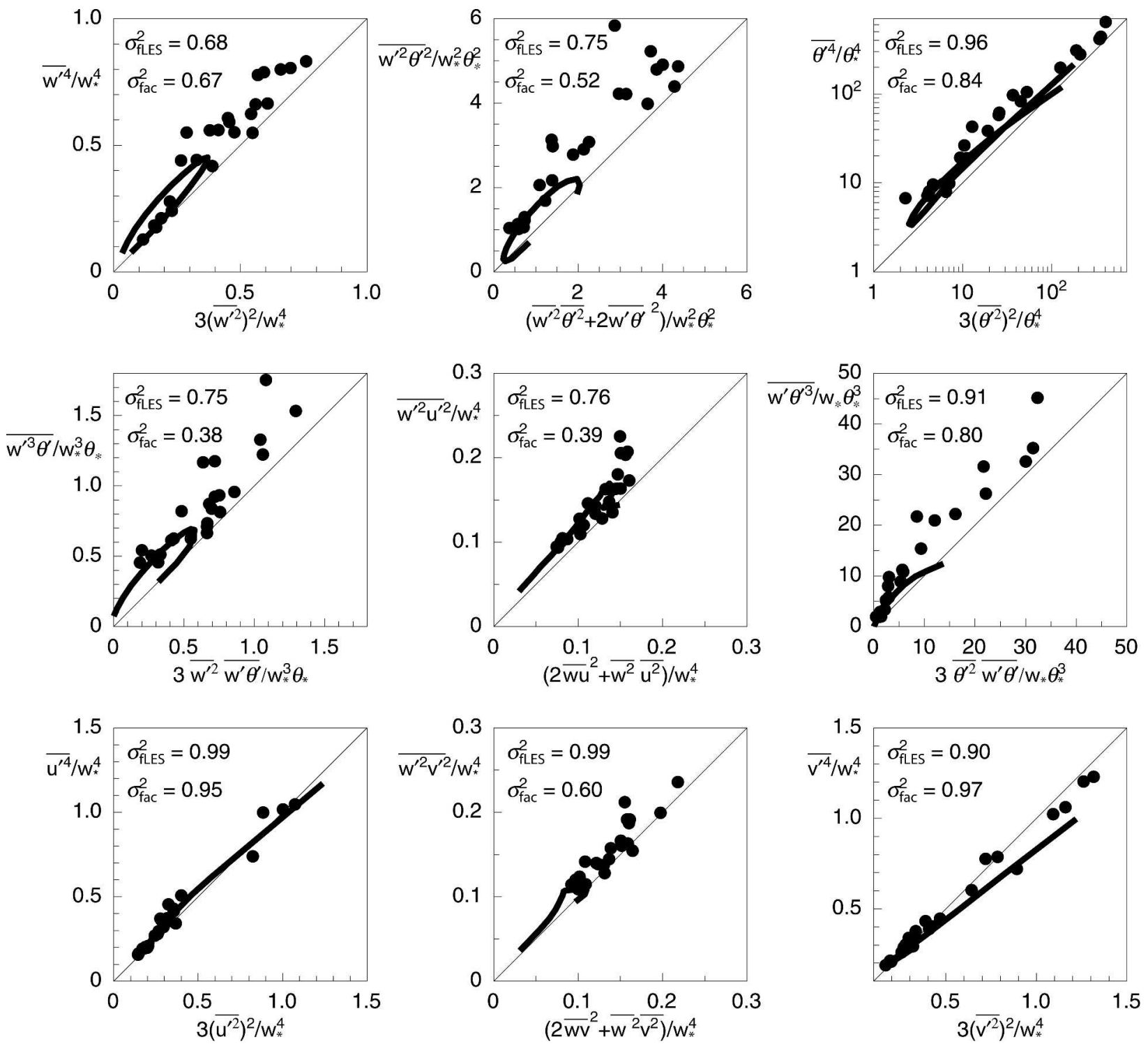

FIG. 1. Aircraft measurements (dots) and LES results (solid lines) of fourth-order moments (ordinates) vs their parameterizations (abscissas) based on the Gaussian assumption. Since the LES data do not include subgrid scales, the range of values for some moments is smaller than that of the aircraft data, especially where large values occur near the surface and near the inversion. Deardorff scaling with $\theta_{*}$ and $w_{*}$ is used for normalization and $\sigma_{f}$ are the explained variances. The Gaussian parameterization systematically underestimates the FOMs. Furthermore, it leads to an ambiguity of the relation between moment and parameterization for different heights in the boundary layer. This is especially seen in the loop feature of the LES results, which are plotted as a continuous line for increasing height.

where

$$
C_{w u}=\frac{\overline{w^{\prime} u^{\prime}}}{{\overline{w^{\prime 2}}}^{1 / 2}{\overline{u^{\prime 2}}}^{1 / 2}}, \quad C_{w v}=\frac{\overline{w^{\prime} v^{\prime}}}{{\overline{w^{\prime 2}}}^{1 / 2}{\overline{v^{\prime 2}}}^{1 / 2}}
$$

are correlation coefficients. The moments (10) and (11) can be formulated in analogy to the first line in (7).

This choice fulfills the conditions given by (i) dimensional analysis, (ii) tensor invariance, (iii) symmetry conditions (Mironov et al. 1999; Abdella and McFarlane 1999), (iv) realizability requirements (Andre et al. 1976), and (v) solution for the 16-delta probability density function model [which is a straightforward generalization of the 3-delta PDF model of Mironov et al. (1999) and GH and the traditional 2-delta PDF model, e.g., Lappen and Randall (2001), see Eq. (13) below].

The parameterizations (2)-(11) shall represent the traditional Millionshchikov hypothesis in the case of 
zero skewness. In the limit of large skewness they shall be derived from an analysis of joint probabilities. We approximate the actual PDF by a simplification that represents the contribution of the coherent structures. In this model, all deviations from the mean of vertical velocity $w$, potential temperature $\theta$, along-stream velocity $u$, and cross-stream velocity $v$ are represented by eight values: $w_{u}$ and $w_{d}$ updraft and downdraft velocities, $\theta_{w}$ and $\theta_{c}$ warm and cold fluctuations, $u_{f}$ and $u_{b}$ forward and backward velocities, and $v_{r}$ and $v_{l}$ left and right velocities. In analogy to a mass-flux representation we can form joint probabilities for these eight quantities: $p_{\text {uwfr }}, p_{\text {defr }}$ are the joint probabilities of warm updraft $w_{u}, \theta_{w}$ and cold downdraft $w_{d}, \theta_{c}$ together with the joint probability $p_{\mathrm{ucfr}}, p_{\mathrm{dwfr}}$ of cold updraft $w_{u}, \theta_{c}$ and warm downdraft $w_{d}, \theta_{w}$ in along-wind forward component $u_{f}$ with deviation to the right $v_{r}$. The joint probabilities $p_{\mathrm{uwfl}}, p_{\mathrm{dcfl}}, p_{\mathrm{ucfl}}, p_{\mathrm{dwfl}}$ are the probabilities of warm updraft, cold downdraft, cold updraft, and warm downdraft of the along-wind forward component with deviation to the left $v_{l}$. The remaining joint probabilities $p_{\text {uwbr }}, p_{\mathrm{dcbr}}, p_{\mathrm{ucbr}}, p_{\mathrm{dwbr}}$, and $p_{\mathrm{uwbl}}, p_{\mathrm{dcbl}}, p_{\mathrm{ucbl}}, p_{\mathrm{dwbl}}$ of along-wind backward streams $u_{b}$ with deviations to the right $v_{r}$ and to the left $v_{l}$, respectively, are defined in the same way.

The 16-delta PDF can be formulated by

$$
\begin{aligned}
P\left(w^{\prime}, \theta^{\prime}, u^{\prime}, v^{\prime}\right)= & {\left[p_{\mathrm{uwfr}} \delta\left(w^{\prime}-w_{u}\right) \delta\left(\theta^{\prime}-\theta_{w}\right)+p_{\mathrm{dcfr}} \delta\left(w^{\prime}-w_{d}\right) \delta\left(\theta^{\prime}-\theta_{c}\right)+p_{\mathrm{ucfr}} \delta\left(w^{\prime}-w_{u}\right) \delta\left(\theta^{\prime}-\theta_{c}\right)\right.} \\
& \left.+p_{\mathrm{dwfr}} \delta\left(w^{\prime}-w_{d}\right) \delta\left(\theta^{\prime}-\theta_{w}\right)\right] \delta\left(u^{\prime}-u_{f}\right) \delta\left(v^{\prime}-v_{r}\right)+\left[p_{\mathrm{uwbr}} \delta\left(w^{\prime}-w_{u}\right) \delta\left(\theta^{\prime}-\theta_{w}\right)\right. \\
& +p_{\mathrm{dcbr}} \delta\left(w^{\prime}-w_{d}\right) \delta\left(\theta^{\prime}-\theta_{c}\right)+p_{\mathrm{ucbr}} \delta\left(w^{\prime}-w_{u}\right) \delta\left(\theta^{\prime}-\theta_{c}\right) \\
& \left.+p_{\mathrm{dwbr}} \delta\left(w^{\prime}-w_{d}\right) \delta\left(\theta^{\prime}-\theta_{w}\right)\right] \delta\left(u^{\prime}-u_{b}\right) \delta\left(v^{\prime}-v_{r}\right)+\left[p_{\mathrm{uwfl}} \delta\left(w^{\prime}-w_{u}\right) \delta\left(\theta^{\prime}-\theta_{w}\right)\right. \\
& +p_{\mathrm{dctl} \delta} \delta\left(w^{\prime}-w_{d}\right) \delta\left(\theta^{\prime}-\theta_{c}\right)+p_{\mathrm{ucfl}} \delta\left(w^{\prime}-w_{u}\right) \delta\left(\theta^{\prime}-\theta_{c}\right) \\
& \left.+p_{\mathrm{dwfl}} \delta\left(w^{\prime}-w_{d}\right) \delta\left(\theta^{\prime}-\theta_{w}\right)\right] \delta\left(u^{\prime}-u_{f}\right) \delta\left(v^{\prime}-v_{l}\right)+\left[p_{\mathrm{uwbl}} \delta\left(w^{\prime}-w_{u}\right) \delta\left(\theta^{\prime}-\theta_{w}\right)\right. \\
& +p_{\mathrm{dcbl}} \delta\left(w^{\prime}-w_{d}\right) \delta\left(\theta^{\prime}-\theta_{c}\right)+p_{\mathrm{ucbl}} \delta\left(w^{\prime}-w_{u}\right) \delta\left(\theta^{\prime}-\theta_{c}\right) \\
& \left.+p_{\mathrm{dwbl}} \delta\left(w^{\prime}-w_{d}\right) \delta\left(\theta^{\prime}-\theta_{w}\right)\right] \delta\left(u^{\prime}-u_{b}\right) \delta\left(v^{\prime}-v_{l}\right),
\end{aligned}
$$

where $\delta(x)$ is the Dirac delta function.

If we integrate the PDF (13) over the horizontal wind components, the 16-delta model coincides with the 4-delta (4-quadrant) mass-flux model of updrafts/ downdrafts and warm/cold areas, where

$$
p_{\mathrm{uwfr}}+p_{\mathrm{uwbr}}+p_{\mathrm{uwfl}}+p_{\mathrm{uwbl}}=p_{\mathrm{uw}}
$$

the probability of warm updraft,

$$
p_{\text {ucfr }}+\mathrm{p}_{\text {uebr }}+p_{\text {ucfl }}+p_{\text {uebl }}=p_{\text {ue }}
$$

the probability of cold updraft,

$p_{\mathrm{dwfr}}+p_{\mathrm{dwbr}}+p_{\mathrm{dwfl}}+p_{\mathrm{dwbl}}=p_{\mathrm{dw}}$

the probability of warm downdraft,

$$
p_{\mathrm{dcfr}}+p_{\mathrm{dcbr}}+p_{\mathrm{dcfl}}+p_{\mathrm{dcbl}}=p_{\mathrm{dc}}
$$

the probability of cold downdraft.

equations with respect to the parameters and substitute the result into the equations for the higher order moments, see GH for details [their Eqs. (4)-(17)]. We find in the limit of large skewness

$$
\begin{aligned}
\overline{w^{\prime 4}} & =S_{w}^{2}{\overline{w^{\prime 2}}}^{2} \\
\overline{w^{\prime 3} \theta^{\prime}} & =S_{w}^{2} \overline{w^{\prime} \theta^{\prime}} \overline{w^{\prime 2}}, \\
\overline{w^{\prime 2} \theta^{\prime 2}} & =S_{w^{\prime}} S_{\theta^{\prime}} \overline{\theta^{\prime}} \overline{w^{\prime 2}}{ }^{1 / 2} \overline{\theta^{\prime 2}}{ }^{1 / 2}, \\
\overline{w^{\prime} \theta^{\prime 3}} & =S_{\theta}^{2} \overline{\bar{w}^{\prime} \theta^{\prime}} \overline{\theta^{\prime 2}}, \\
\overline{\theta^{\prime 4}} & =S_{\theta}^{2} \overline{\theta^{\prime 2}}{ }^{2} .
\end{aligned}
$$

In analogy, the moments involving the horizontal wind components $u$ and $v$ are formed. Comparing (19)-(23) with (2)-(7) and the corresponding equations for the $u$
Randall 2001).

By comparison with the Millionshchikov assumption
[Eq. (1)] the first requirement (Gaussian behavior in

By straightforward algebraic calculation of the moment with the help of the PDF (13) we find the moments in terms of PDF parameters. Next we solve the lowest 

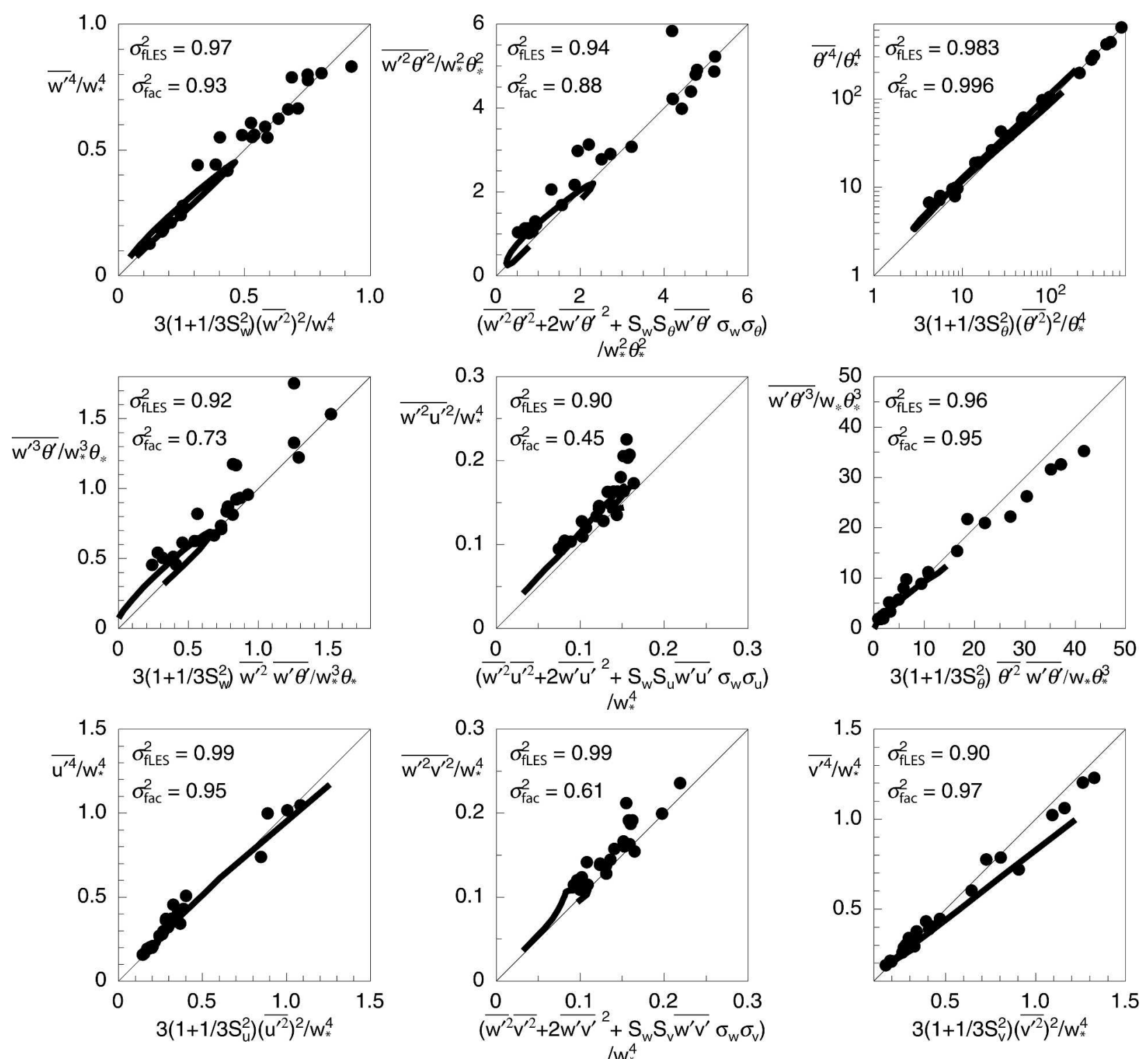

FIG. 2. Aircraft measurements (dots) and LES results (solid lines) of fourth-order moments (ordinates) vs the new parameterizations Eqs. (2)-(11) (abscissas); $\sigma_{f}$ are the explained variances. Since the LES data do not include subgrid scales, the range of values for some moments is smaller than that of the aircraft data, especially where large values occur near the surface and near the inversion.

and $v$ components with (8)-(11), we find in the limit of large skewness

$$
d_{i}=\frac{1}{3}(i=3, \ldots, 8,9), \quad d_{i}=1(i=7,10,11) .
$$

For $i=3, \ldots, 6$, Eqs. (18) and (24) coincide with the coefficients obtained for the 3-delta PDF model in GH. Substitution of the nondimensional coefficients $a_{i}$ (18) and $d_{i}(24)$ into Eqs. (2)-(12) gives the universal closure model, which describes the Gaussian convective regime in the limit of small skewness, and strongly non-
Gaussian skewed turbulence in the limit of large skewness. It presents a linear interpolation between these two limiting conditions.

\section{Testing of new FOM versus data and LES}

In Fig. 2 we compare the refined Millionshchikov parameterizations given by Eqs. (2)-(11) versus measurements and LES data. Based on the explained variance, all moments show a better agreement for both aircraft measurements and LES data, except the fourth- 
order moment of the across-wind component $\overline{v^{\prime 4}}$, which is virtually unchanged. Furthermore, the ambiguity in the relation for different heights has almost disappeared, seen as the nearly collapsed loop in the LES data. The parameterization becomes single valued for the moments $\overline{w^{\prime 4}}, \overline{\theta^{\prime 4}}$ and $\overline{w^{\prime} \theta^{\prime 3}}$, and reduces for $\overline{w^{\prime 3} \theta^{\prime}}$ of the LES results.

We also tested the parameterizations (2)-(11) with LES simulation data of 4 and $0 \mathrm{~m} \mathrm{~s}^{-1}$ geostrophic wind speed while all other boundary layer parameters were unchanged. These results also confirm the unsuitability of the Millionshchikov hypothesis and show a significantly better agreement with our new formulations.

\section{Realizability of the new parameterizations}

The drawback of the Millionshchikov closure for convective conditions becomes also evident from the analysis of measurements and LES data. The analysis shows that statistics (SOM, TOM, and FOM) do not satisfy the realizability conditions (Andre et al. 1976)

$$
{\overline{a^{\prime} b^{\prime} c^{\prime}}}^{2} \leq \min \left\{\begin{array}{l}
\overline{a^{\prime 2}}\left(\overline{b^{\prime 2} c^{\prime 2}}-\overline{b^{\prime 2}} \cdot \overline{c^{\prime 2}}\right), \\
\overline{b^{\prime 2}}\left(\overline{a^{\prime 2} c^{\prime 2}}-\overline{a^{\prime 2}} \cdot \overline{c^{\prime 2}}\right), \\
\overline{c^{\prime 2}}\left(\overline{a^{\prime 2} b^{\prime 2}}-\overline{a^{\prime 2}} \cdot \overline{b^{\prime 2}}\right),
\end{array}\right.
$$

where $a^{\prime}, b^{\prime}, c^{\prime}, d^{\prime}$ are arbitrary fluctuations of $u^{\prime}, v^{\prime}$, $w^{\prime}, \theta^{\prime}$.

The measurements and LES statistics satisfy the realizability conditions (25) as shown for the potential temperature $\theta$ in Fig. 3, which shows the realizability condition for $a=b=c=\theta$

$$
K_{\theta} \geq 1+S_{\theta}^{2},
$$

where $K_{\theta}=\overline{\theta^{\prime 4}} /{\overline{\theta^{\prime 2}}}^{2}$ is the kurtosis and $S_{\theta}$ the skewness. If the kurtosis is calculated by the Millionshchikov closure hypothesis (1) we have $K_{\theta}=3$ (plotted by the horizontal line in Fig. 3), the realizability condition (26) would be violated for $S_{\theta}>\sqrt{2}$. The closure (3) is in agreement with condition (26), since the $K_{\theta}=3(1+$ $\left.1 / 3 S_{\theta}^{2}\right) \geq 1+S_{\theta}^{2}$. The other realizability conditions, for example, $K_{\theta} \geq 1+\overline{w^{\prime} \theta^{\prime 2}}{ }^{2} /\left(\overline{w^{\prime 2}}{\overline{\theta^{\prime 2}}}^{2}\right)$ following from (25) for $a=b=\theta$ and $c=w$ are also satisfied by the new FOM parameterizations. Substituting the closure (3) into the lhs, we find $S_{\theta}^{2} \geq \overline{w^{\prime} \theta^{\prime 2}} /\left(\overline{w^{\prime 2}} \frac{\theta^{\prime 2}}{2}\right)-2$. This condition is satisfied since $S_{\theta}^{2} \geq 0$ and $\overline{w^{\prime} \theta^{\prime 2}} /\left(\overline{w^{\prime 2}} \overline{\theta^{\prime 2}}{ }^{2}\right)$ $-2 \leq-1$.

\section{Discussion and conclusions}

The measurements and LES data provide counterexamples for the Millionshchikov hypothesis. The results

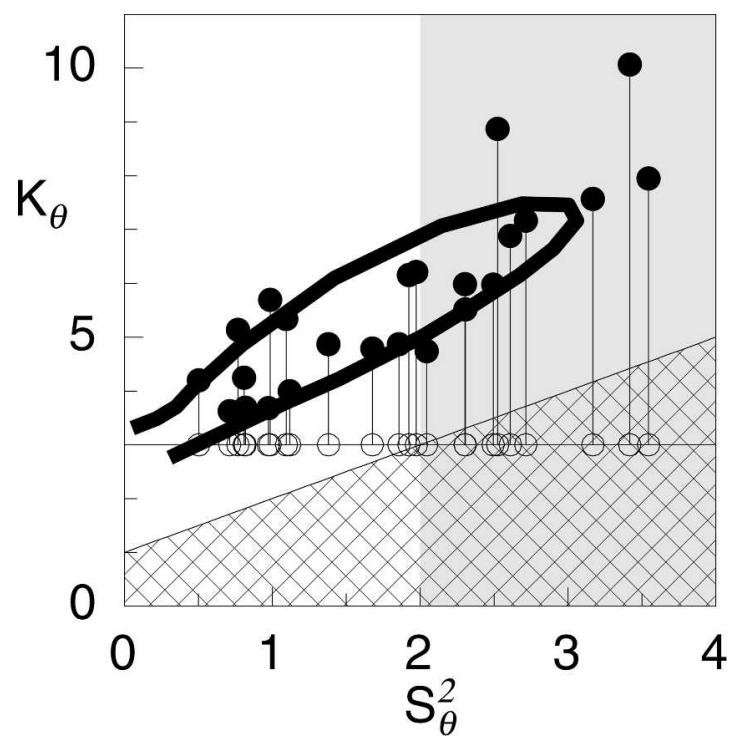

FIG. 3. Kurtosis vs the square of the skewness for the aircraft data (dots) and the LES results (solid lines). Hatching marks the nonrealizable area. The data with $S^{2}>2$ (light gray area) are not realizable with the Gaussian parameterization.

presented in Figs. 1 and 2 show that overall at CBL conditions, the Millionshchikov hypothesis is not well suited for parameterization of FOM involving the vertical velocity and temperature.

In the range of parameters studied, the parameterizations involving horizontal velocity fluctuations have little skewness and are close to Gaussian. Here, the Millionshchikov hypothesis remains valid for these moments, and the simpler parameterization (1) may be used.

Implementation of the parameterizations (2)-(11) into the transport terms of the TOM equations shows that the redistributions of the FOMs not only depend on the gradients of the SOMs, as the Millionshchikov hypothesis states, but also on the gradients of the TOMs as well.

Since the parameterizations (2)-(11) express the FOM in terms of second-order moments and TOM, the minimal closure model for convective conditions should be the TOM closure model.

The TOM model can be simplified, for example, assuming stationarity of the TOM equations (cf. Canuto and Dubovikov 1998). It can be reduced to a simple SOM equations model.

The universal model may also be valid for moisture and passive scalar fluctuations, which shall be examined in further studies.

\section{REFERENCES}

Abdella, K., and N. McFarlane, 1999: Reply. J. Atmos. Sci., 56, 3482-3483. 
Alberghi, S., A. Maurizi, and E. Tampieri, 2002: Reply. J. Appl. Meteor., 41, 885-889.

Andre, J. C., G. De Moor, P. Lacarrère, and R. du Vachat, 1976: Turbulence approximation for inhomogeneous flows: Part I. The clipping approximation. J. Atmos. Sci., 33, 476-481.

Canuto, V. M., and M. Dubovikov, 1998: Stellar turbulent convection: Theory. Astrophys. J., 493, 834-847.

—, F. Minotti, C. Ronchi, R. M. Ypma, and O. Zeman, 1994: Second-order closure PBL model with new third-order moments: Comparison with LES data. J. Atmos. Sci., 51, 16051618.

- Y. Cheng, and A. Howard, 2001: New third-order moments for the convective boundary layer. J. Atmos. Sci., 58, 11691172.

Gryanik, V. M., and J. Hartmann, 2002: A turbulence closure for the convective boundary layer based on a two-scale mass-flux approach. J. Atmos. Sci., 59, 2729-2744.

Hartmann, J., and Coauthors, 1999: Artic Radiation and Turbulence Interaction Study. Berichte zur Polarforschung 305, Alfred Wegener Institute for Polar and Marine Sciences, $81 \mathrm{pp}$.

Ilyushin, B. B., and A. F. Kurbatskii, 1997: Modeling of turbulent transport in PBL with third-order moments. Proc. 11th Symp. on Turbulent Shear Flows, Vol. 2, Grenoble, France, 20192021.

Lappen, C. L., and D. A. Randall, 2001: Toward a unified param- eterization of the boundary layer and moist convection. Part I: A new type of mass-flux model. J. Atmos. Sci., 58, 20212036.

Losch, M., 2004: On the validity of the Millionshchikov quasinormality hypothesis for open-ocean deep convection. Geophys. Res. Lett., 31, L23301, doi:10.1029/2004GL021412.

Millionshchikov, M. D., 1941: On the theory of homogeneous isotropic turbulence. Dokl. Akad. Nauk SSSR, 32, 611-614.

Mironov, D. V., V. M. Gryanik, V. N. Lykossov, and S. S. Zilitinkevich, 1999: Comments on "A new second-order turbulence closure scheme for the planetary boundary layer." $J$. Atmos. Sci., 56, 3478-3481.

Moeng, C.-H., and D. A. Randall, 1984: Problems in simulating the stratocumulus-topped boundary layer with a third-order closure model. J. Atmos. Sci., 41, 1588-1600.

Monin, A. S., and A. M. Yaglom, 1971: Statistical Fluid Mechanics. Vol. 1, MIT Press, 769 pp.

Raasch, S., and M. Schröter, 2001: PALM-A large-eddy simulation model performing on massively parallel computers. $\mathrm{Me}$ teor. Z., 10, 363-372.

Zilitinkevich, S., V. M. Gryanik, V. N. Lykossov, and D. V. Mironov, 1999: Third-order transport and nonlocal turbulence closures for convective boundary layers. J. Atmos. Sci., 56, 3463-3477. 